N. NINOMIYA

KŌDAI MATH. SEM. REP.

27 (1976), 300-307

\title{
A NOTE ON THE TRANSFINITE DIAMETER
}

\section{To Professor Yûsaku Komatu on the occasion of his 60th birthday}

\author{
By NoBUyUKI NINOMIYA
}

In this note, we shall try to extend the notion of the transfinite diameter taken with respect to a symmetric kernel to the case when a kernel is not always symmetric. In a locally compact Hausdorff space, let $K(P, Q)$ be a continuous function in $P$ and $Q,+\infty$ for $P=Q$, finite for $P \neq Q$ and symmetric: $K(P, Q)=$ $K(Q, P)$. For any compact set $F$ of $\Omega$ containing an infinite number of points, put

$$
W_{n}(F)=\inf \frac{\sum_{i<j} K\left(P_{\imath}, P_{\jmath}\right)}{\left(\frac{n}{2}\right)},
$$

where inf is taken with respect to pairs of $n$ distinct points $P_{1}, P_{2}, \cdots, P_{n}$ of $F$. Owing to the hypothesis for the function $K$ to be continuous, the inf is attained by a pair of $n$ distinct points $Q_{1}, Q_{2}, \cdots, Q_{n}$ of $F$, and so the inf can be replaced by min. $K$ being symmetric, we have

$$
W_{n}(F)=\min \frac{\sum_{\imath \neq j} K\left(P_{\imath}, P_{\jmath}\right)}{n(n-1)} .
$$

An important fact is that $W_{n}(F)$ increases with $n$. Any compact set $F$ is said to be of $K$-transfinite diameter zero if

$$
W(F)=\lim _{n \rightarrow+\infty} W_{n}(F)=+\infty,
$$

and said to be of $K$-transfinite diameter positive if $W(F)<+\infty$.

The notion of the transfinite diameter was obtained for the first time by Fekete for sets on the plane (to see [1]), and next by Pólya-Szegö for sets in the ordinary space (to see [6]). According to Pólya-Szegö, for any compact set $F$ of the $m$-dimensional Euclidean space $R^{m}(m \geqq 3)$ containing an infinite number of points and $n$ variable points $P_{1}, P_{2}, \cdots, P_{n}$ of $F$, put

$$
\left\{D_{n}(F)\right\}^{\alpha-m}=W_{n}(F)=\min \frac{\sum_{i<j} \overline{P_{i} P_{j}^{\alpha-m}}}{\left(\frac{n}{2}\right)} \quad(0<\alpha<m) .
$$

Received May 7, 1974. 
Then, $D_{n}(F)$ decreases when $n$ increases. When we put

$$
\lim _{n \rightarrow+\infty} D_{n}(F)=D(F) \quad \text { and } \quad \lim _{n \rightarrow+\infty} W_{n}(F)=W(F),
$$

we have naturally

$$
0 \leqq D(F)<+\infty \quad \text { and } \quad 0<W(F) \leqq+\infty .
$$

$D(F)$ is called the $\alpha$-ordered transfinite diameter of $F$. According to PólyaSzegö and Frostman, $W(F)$ is equal to the minimum of the $\alpha$-ordered energy integral of positive measures $\mu$ supported by $F$ with total mass 1 (to see [6], p. 15 and [3], p. 46):

$$
W(F)=\min _{\mu} \iint \overline{P Q}^{\alpha-m} d \mu(Q) d \mu(P) .
$$

This fact is assured to be also valid in the case of $K(P, Q)$ and $W(F)$ as stated on the beginning.

The following theorem is well-known as the Evans' theorem (to see [2]): Given any compact set $F$ in $R^{m}(m \geqq 3)$ of Newtonian transfinite diameter zero (equivalent to say "of Newtonian capacity zero"), there exists a positive measure $\mu$ supported by $F$ with total mass 1 whose potential

$$
U^{\mu}(P)=\int \overline{P Q}^{2-m} d \mu(Q)
$$

is $+\infty$ at each point $P$ of $F$. This theorem is assured to be also valid for the potential taken with respect to a kernel $K(P, Q)$ as stated on the beginning (to see [5]).

The hypothesis of continuity and symmetricity for a kernel $K$ seems to play an essential role in the statements so far discussed on the transfinite diameter, the capacity and the Evans' theorem. In this note we are going to introduce a notion of the transfinite diameter taken with respect to a non-symmetric kernel and to extend the Evans' theorem.

In a locally compact Hausdorff space $\Omega$, let $K(P, Q)$ be a lower semi-continuous function in $P$ and $Q$, may be $+\infty$ for $P=Q$, always finite for $P \neq Q$ and bounded from above for $P$ and $Q$ belonging to disjoint compact sets respectively." It is not assumed for $K$ to be symmetric. For any compact set $F$ of $\Omega$ and a non-negative number $t$, consider the quantity*)

$$
W_{n}(F)=\inf \frac{\sum_{i=j} K\left(P_{\imath}, P_{\jmath}\right)+t \sum_{i>\jmath} K\left(P_{\imath}, P_{\jmath \jmath}\right)}{n(n-1)},
$$

where inf is taken with respect to pairs of $n$ variable points $P_{1}, P_{2}, \cdots, P_{n}$ of $F$, admitted to be overlapping. $W_{n}(F)$ will be finite or positively infinite. Owing

*) Nakai has studied the transfinite diameter in his paper (to see [4], p. 222). His idea is the case of $t=0$ in the quantity. The Evans' theorem is obtained there, but no relation between the transfinite diameter and the capacity is discussed. 
to the hypothesis for the function $K$ to be lower semi-continuous, the inf is attained by a pair of $n$ points $Q_{1}, Q_{2}, \cdots, Q_{n}$ of $F$ which may be overlapping, and so the inf can be replaced by min. $W_{n}(F)$ coincided with one as stated on the beginning if $K$ is symmetric and $t=1$. Then, there holds

THEOREM 1. For any compact set $F$ of $\Omega$ and a non-negative number $t$, $W_{n}(F)$ increases with $n$.

Proof. First of all, we should like to show that the value of $W_{n}(F)$ is really attained by a pair $\left(Q_{1}, Q_{2}, \cdots, Q_{n}\right)$ of $n$ points of $F$. Let $\left(P_{1 k}, P_{2 k}, \cdots, P_{n k}\right)$ be a sequence of pairs of $n$ points of $F$, admitted to be overlapping, such that

$$
\frac{\sum_{i<j} K\left(P_{i k}, P_{j k}\right)+t \sum_{i>j} K\left(P_{i k}, P_{j k}\right)}{n(n-1)} \downarrow W_{n}(F)
$$

when $k \rightarrow+\infty$. Let $Q_{1}, Q_{2}, \cdots$, and $Q_{n}$ be the limiting points of $P_{1 k}, P_{2 k}, \cdots$ and $P_{n k}$ respectively, if necessary, by extracting their suitable subsequences. The function $K$ being lower semi-continuous and $t$ being non-negative, we have

$$
\begin{aligned}
W_{n}(F) & \leqq \frac{\sum_{i<j} K\left(Q_{\imath}, Q_{\jmath}\right)+t \sum_{i>j} K\left(Q_{i}, Q_{\jmath}\right)}{n(n-1)} \\
& \leqq \lim _{k \rightarrow+\infty} \frac{\sum_{i<j} K\left(P_{i k}, P_{j k}\right)+t \sum_{i>j} K\left(P_{i k}, P_{j k}\right)}{n(n-1)} \\
& =W_{n}(F),
\end{aligned}
$$

and so

$$
W_{n}(F)=\frac{\sum_{i<j} K\left(Q_{\imath}, Q_{\jmath}\right)+t \sum_{i>j} K\left(Q_{\imath}, Q_{\jmath}\right)}{n(n-1)},
$$

whose right hand side will be denoted by

$$
\frac{v_{n}}{n(n-1)} \text {. }
$$

Although the pair $\left(Q_{1}, Q_{2}, \cdots, Q_{n}\right)$ outght to be written strictly by $\left(Q_{1}^{n}, Q_{2}^{n}, \cdots, Q_{n}^{n}\right)$, we shall go ahead without doing so, since $n$ is fixed. Some of $Q_{1}, Q_{2}, \cdots$ and $Q_{n}$ may be overlapping. Then, we have $n$ following inequalities :

$$
\begin{aligned}
v_{n}= & \sum_{\jmath=2}^{n} K\left(Q_{1}, Q_{j}\right)+t \sum_{i=2}^{n} K\left(Q_{i}, Q_{1}\right)+\sum_{\substack{i<j j \\
i, j \neq 1}} K\left(Q_{i}, Q_{\jmath}\right)+t \sum_{\substack{i>\jmath \\
i, j \neq 1}} K\left(Q_{\imath}, Q_{\jmath}\right) \\
\geqq & \sum_{j=2}^{n} K\left(Q_{1}, Q_{\jmath}\right)+t \sum_{i=2}^{n} K\left(Q_{\imath}, Q_{1}\right)+v_{n-1}, \\
v_{n}= & \sum_{\jmath=3}^{n} K\left(Q_{2}, Q_{\jmath}\right)+t \sum_{i=3}^{n} K\left(Q_{\imath}, Q_{2}\right)+K\left(Q_{1}, Q_{2}\right)+t K\left(Q_{2}, Q_{1}\right) \\
& +\sum_{\substack{i<j \\
i, j \neq 2}} K\left(Q_{i}, Q_{j}\right)+t \sum_{\substack{i, j \\
\imath, j \neq 2}} K\left(Q_{\imath}, Q_{\jmath}\right)
\end{aligned}
$$




$$
\begin{aligned}
& \geqq \sum_{\jmath=3}^{n} K\left(Q_{2}, Q_{\jmath}\right)+t \sum_{\imath=3}^{n} K\left(Q_{\imath}, Q_{2}\right)+K\left(Q_{1}, Q_{2}\right)+t K\left(Q_{2}, Q_{1}\right)+v_{n-1}, \\
& v_{n} \geqq \sum_{\jmath=4}^{n} K\left(Q_{3}, Q_{\jmath}\right)+t \sum_{\imath=4}^{n} K\left(Q_{\imath}, Q_{3}\right) \\
& +K\left(Q_{1}, Q_{3}\right)+K\left(Q_{2}, Q_{3}\right)+t K\left(Q_{3}, Q_{1}\right)+t K\left(Q_{3}, Q_{2}\right)+v_{n-1}, \\
& v_{n} \geqq K\left(Q_{n-1}, Q_{n}\right)+t K\left(Q_{n}, Q_{n-1}\right)+\sum_{\imath=1}^{n-2} K\left(Q_{\imath}, Q_{n-1}\right)+t \sum_{\jmath=1}^{n-2} K\left(Q_{n-1}, Q_{\jmath}\right)+v_{n-1}
\end{aligned}
$$

and finally

$$
v_{n} \geqq \sum_{\imath=1}^{n-1} K\left(Q_{\imath}, Q_{n}\right)+t \sum_{\jmath=1}^{n-1} K\left(Q_{n}, Q_{\jmath}\right)+v_{n-1} .
$$

Summing up these inequalities, we have

$$
n v_{n} \geqq v_{n}+v_{n}+n v_{n-1} .
$$

Thus, we have

$$
W_{n-1}(F) \leqq W_{n}(F) .
$$

Definition. Given any compact set $F$ of $\Omega$ and a non-negative number $t, F$ is said to be of $(K, t)$-transfinite diameter zero if

$$
W(F)=\lim _{n \rightarrow+\infty} W_{n}(F)=+\infty,
$$

and said to be of $(K, t)$-transfinite diameter positive if $W(F)<+\infty$.

Consider the potential and the energy integral of positive measures $\mu$ taken with respect to a kernel $K$ and its adjoint kernel $\check{K}$ :

and

$$
\begin{aligned}
& K(P, \mu)=\int K(P, Q) d \mu(Q) \\
& K(\mu, P)=\int \check{K}(P, Q) d \mu(Q)=\int K(Q, P) d \mu(Q)
\end{aligned}
$$

$$
K(\mu, \mu)=\iint K(P, Q) d \mu(Q) d \mu(P)=\iint \check{K}(P, Q) d \mu(Q) d \mu(P) .
$$

Then, there holds

THEOREM 2. Let $t$ be a non-negative number. Given any compact set $F$ of $\Omega$ of $(K, t)$-transfinite diameter zero, there exists a positive measure $\mu$ supported by $F$ with total mass 1 such that

and

$$
K(P, \mu)+t K(\mu, P)=+\infty
$$

$$
K(\mu, P)+t K(P, \mu)=+\infty
$$

at each point $P$ of $F$. 
Proof. Once for all, denote by $\left(Q_{1}^{n}, Q_{2}^{n}, \cdots, Q_{n}^{n}\right)$ a pair of $n$ points of $F$ where the value of $W_{n}(F)$ is attained. They are admitted to be overlapping. Then, we have for any point $P$ of $F$

$$
\begin{aligned}
W_{n}(F) & \leqq W_{n+1}(F) \\
& \leqq \frac{\sum_{i<j} K\left(Q_{i}^{n+1}, Q_{j}^{n+1}\right)+t \sum_{i>j} K\left(Q_{i}^{n+1}, Q_{j}^{n+1}\right)}{(n+1) n} \\
& \leqq \frac{\sum_{j=1}^{n} K\left(P, Q_{j}^{n}\right)+t \sum_{i=1}^{n} K\left(Q_{i}^{n}, P\right)+\sum_{i<j} K\left(Q_{i}^{n}, Q_{j}^{n}\right)+t \sum_{i>j} K\left(Q_{i}^{n}, Q_{j}^{n}\right)}{(n+1) n} \\
& \leqq \frac{\sum_{j=1}^{n} K\left(P, Q_{j}^{n}\right)+t \sum_{i=1}^{n} K\left(Q_{i}^{n}, P\right)+n(n-1) W_{n}(F)}{(n+1) n} .
\end{aligned}
$$

Accordingly, there holds

$$
2 W_{n}(F) \leqq \frac{1}{n}\left\{\sum_{i=1}^{n} K\left(P, Q_{i}^{n}\right)+t \sum_{i=1}^{n} K\left(Q_{i}^{n}, P\right)\right\},
$$

and similarly holds

$$
2 W_{n}(F) \leqq \frac{1}{n}\left\{\sum_{i=1}^{n} K\left(Q_{i}^{n}, P\right)+t \sum_{i=1}^{n} K\left(P, Q_{i}^{n}\right)\right\} .
$$

Let $k$ be any positive number and $W_{n}(F)>2^{k}$ for some large number $n$. Let $\mu_{k}$ be the measure with the mass $n^{-1} \cdot 2^{-k}$ at each point $Q_{i}^{n}$. It is a positive measure supported by $F$ with total mass $2^{-k}$. Evidently, we have

and

$$
2 \leqq K\left(P, \mu_{k}\right)+t K\left(\mu_{k}, P\right)
$$

$$
2 \leqq K\left(\mu_{k}, P\right)+t K\left(P, \mu_{k}\right)
$$

at each point $P$ of $F$. Then, for the measure

$$
\mu=\sum_{k=1}^{+\infty} \mu_{k}
$$

there hold

$$
K(P, \mu)+t K(\mu, P)=+\infty
$$

and

$$
K(\mu, P)+t K(P, \mu)=+\infty
$$

at each point $P$ of $F$.

THEOREM 3. Let $F$ be any compact set of $\Omega$ and $t$ a positive number. $F$ is of $(K, t)$-transfinite diameter positive if and only if there exist positive measures of finite K-energy supported by $F$.

Proof. Let $\mu$ be a positive measure of finite $K$-energy supported by $F$ with total mass 1 . For $n$ arbitrary points $P_{1}, P_{2}, \cdots, P_{n}$ of $F$, we have 


$$
W_{n}(F) \leqq \frac{\sum_{\imath<j} K\left(P_{\imath}, P_{\jmath}\right)+t \sum_{i>j} K\left(P_{\imath}, P_{\jmath}\right)}{n(n-1)},
$$

hence, regarding the right hand side as a function in $n$ variable points $P_{1}, P_{2}$, $\cdots, P_{n}$ and intergrating the inequality by the positive measure

$$
d \mu\left(P_{1}\right) d \mu\left(P_{2}\right) \cdots d \mu\left(P_{n}\right)
$$

whose total mass is one, we have

$$
W_{n}(F)=\frac{\left(\begin{array}{l}
n \\
2
\end{array}\right) K(\mu, \mu)+t\left(\begin{array}{l}
n \\
2
\end{array}\right) K(\mu, \mu)}{n(n-1)}=\frac{(1+t) K(\mu, \mu)}{2}<+\infty
$$

and so

$$
2 W(F) \leqq(1+t) K(\mu, \mu)<+\infty .
$$

In the next, suppose that $W(F)<+\infty$. We are going to find a positive measure $\mu$ of finite $K$-energy supported by $F$ with total mass 1 . Take $n$ points $Q_{1}, Q_{2}$, $\cdots, Q_{n}$ of $F$ such that

$$
W_{n}(F)=\frac{\sum_{i \leq j} K\left(Q_{\imath}, Q_{\jmath}\right)+t \sum_{i>\jmath} K\left(Q_{\imath}, Q_{\jmath}\right)}{n(n-1)} .
$$

Let $\mu_{n}$ be the measure with $1 / n$ at each $Q_{\imath}(\imath=1,2, \cdots, n)$. It is a positive measure supported by $F$ with total mass 1 . Suppose that $\left\{\mu_{n}\right\}$ converges vaguely to a measure $\mu$, if necessary, by extracting its suitable subsequence. Then, $\mu$ is a positive measure supported by $F$ with total mass 1 . Let $\left\{f_{k}(P, Q)\right\}$, $k=1,2, \cdots$, be a sequence of finite and continuous functions that increases monotoneously to $K(P, Q)$ and $C$ a positive number such that $f_{1}(P, Q)+C>0$ for all the points $P$ and $Q$ of $F$. Putting

$$
K^{\prime}(P, Q)=K(P, Q)+C \quad \text { and } \quad f_{k}^{\prime}(P, Q)=f_{k}(P, Q)+C,
$$

we have

$$
\begin{aligned}
W_{n}(F)+\frac{1+t}{2} C & =\frac{\sum_{i \leq j} K^{\prime}\left(Q_{\imath}, Q_{\jmath}\right)+t \sum_{i>j} K^{\prime}\left(Q_{\imath}, Q_{\jmath}\right)}{n(n-1)} \\
& \geqq \min (1, t) \frac{\sum_{\imath \neq \jmath} K^{\prime}\left(Q_{\imath}, Q_{\jmath}\right)}{n(n-1)} \\
& \geqq \min (1, t) \frac{\sum_{\imath \neq j} f_{k}^{\prime}\left(Q_{\imath}, Q_{\jmath}\right)}{n(n-1)} \\
& \geqq \min (1, t) \frac{\sum_{\imath, j} f_{k}^{\prime}\left(Q_{\imath}, Q_{\jmath}\right)-n \cdot \max _{F} f_{k}^{\prime}(P, P)}{n(n-1)} \\
& =\min (1, t) \frac{n}{n-1}\left\{\sum_{\imath, \jmath} f_{k}^{\prime}\left(Q_{\imath}, Q_{\jmath}\right) \frac{1}{n^{2}}-\frac{1}{n} \max f_{k}^{\prime}(P, P)\right\} \\
& =\min (1, t) \frac{n}{n-1}\left\{\iint f_{k}^{\prime}(P, Q) d \mu_{n}(Q) d \mu_{n}(P)-\frac{1}{n} \max _{F} f_{k}^{\prime}(P, P)\right\} .
\end{aligned}
$$


Fixing $k$ and making $n \rightarrow+\infty$, we have

$$
W_{n}(F)+\frac{1+t}{2} C \geqq \min (1, t) \iint f_{k}^{\prime}(P, Q) d \mu(Q) d \mu(P),
$$

hence, making $k \rightarrow+\infty$, we have

$$
W(F)+\frac{1+t}{2} C \geqq \min (1, t) \iint K^{\prime}(P, Q) d \mu(Q) d \mu(P),
$$

that is,

$$
K(\mu, \mu) \leqq \frac{2 W(F)+(1+t) C}{2 \min (1, t)}-C<+\infty .
$$

Definition. Any compact set $F$ of $\Omega$ is said to be of $K$-capacity positive if there exist positive measures $\mu$ supported by $F$ whose potential $K(P, \mu)$ is bounded from above on all the compact sets of $\Omega$. Owing to the hypothesis for a kernel $K$, a compact set $F$ is of $K$-capacity zero if and only if

$$
\sup _{F} K(P, \mu)=+\infty
$$

for every positive measure $\mu$ supported by $F$.

Definition. A kernel $K$ is said to satisfy the continuity principle if there holds the following property: Given any positive measure $\mu$ with compact support $F$ and its potential $K(P, \mu)$, every continuous point of $K(P, \mu)$ as a function on $F$ is also a continuous point of $K(P, \mu)$ as a function in $\Omega$.

The following is an important fact in that it expresses a relation between the transfinite diameter and the capacity.

Proposition. Any compact set $F$ of K-capacity positive always supports positive measures of finite K-energy. The converse is also correct if a kernel $K$ satisfies the continuity principle.

In fact, given any compact set $F$ of $K$-capacity positive, take a positive measure $\mu$ supported by $F$ whose potential $K(P, \mu)$ is bounded from above on all the compact sets of $\Omega$. It is evident that $\mu$ is of finite $K$-energy. Conversely, suppose that a kernel $K$ satisfies the continuity principle and that, given any compact set $F$ of $\Omega$, a positive measure $\mu$ supported by $F$ is of finite $K$-energy. Then, the set

$$
\{P ; K(P, \mu)=+\infty\}
$$

being of $\mu$-mass zero, there exists a restricted measure $\mu^{\prime}$ ( $\left.\neq 0\right)$ of $\mu$ such that $K(P, \mu)$ is finite and continuous as a function on the support of $\mu^{\prime}$. By the lower semi-continuity of the potential and the continuity principle, it is easily seen that $K\left(P, \mu^{\prime}\right)$ is finite and continuous at each point of the support of $\mu^{\prime}$. Hence, by the hypothesis for a kernel $K$, it is also seen that $K\left(P, \mu^{\prime}\right)$ is bounded from above on all the compact sets of $\Omega$. 
When a kernel $K$ satisfies the continuity principle, any compact set $F$ of $K$-capacity zero supports no positive measures of finite $K$-energy. Therefore, such a compact set $F$ is always of $(K, t)$-transfinite diameter zero for any positive number $t$. Thus, we can extend the Evans' theorem in the following form.

THEOREM 4. Suppose that a kernel $K$ satisfies the continunty principle. Let $F$ be any compact set of $K$-capacity zero of $\Omega$. Given any positive number $t$, there exists a positive measure $\mu$ supported by $F$ (naturally depending upon $t$ ) such that

$$
K(P, \mu)+t K(\mu, P)=+\infty
$$

and

at each point $P$ of $F$.

$$
K(\mu, P)+t K(P, \mu)=+\infty
$$

QUESTION. Is the above theorem also valid for $t=0$ ?

\section{REFERENCES}

[1] Fekete, M., Über die Verteilung der Wurzeln bei gewissen algebraischen Gleichungen mit ganzzahligen Koeffizienten, Math. Zeitschr., 17 (1923), 228249.

[2] Evans, G.C., Potentials and positively infinite singularities of harmonic functions, Monatsch. Math. Phys., 43 (1936), 419-424.

[3] Frostman, O., Potentiel d'équilibre et capacité des ensembles avec quelques applications à la théorie des fonctions, Medd. Lund Univ. Mat. Sem., 3 (1935), 1-118.

[4] NAKAI, M., Green potential of Evans' type on Royden's compactification of a Riemann surface, Nagoya Math. J., 24 (1964), 205-239.

[5] Ninomiya, N., Sur le principe de continuité dans la théorie du potentiel, J. Inst. Polytech., Osaka City Univ., 8 (1957), 51-56.

[6] Pólya, G. ANd Szegö, G., Über den transfiniten Durchmesser (KapazitätsKonstante) von ebenen und räumlichen Punktmengen, J. Crelle, 165 (1931), $4-49$. 